\title{
Typewriting Dynamics: What Distinguishes Simple From Complex Writing Tasks?
}

\author{
Sebastian Wallot and Joachim Grabowski
}

\section{QUERY SHEET}

This page lists questions we have about your paper. The numbers displayed at the left can be found in the text of the paper for reference. In addition, please review your paper as a whole for correctness.

Q1. Au: Please clarify "under review." Has O'Brien et al. been accepted for publication?

Q2. Au: Define KPI. Also, "copy-typing" is spelled "copytyping" in Figures 1 through 7; Figure 8 and the main text of the article spell it "copy-typing."

Q3. Au: Spell out KPI

Q4. Au: Should this be spelled principle or principal?

Q5. Au: Spell out KPI, PSD, CWT.

Q6. Au: Please clarify "under review." Has O'Brien et al. been accepted for publication?

Q7. Au: Please provide all author names for Andersson et al.

Q8. Au: Please clarify "under review." Has O'Brien et al. been accepted for publication?

Q9. Au: Please check page range for Van Orden (2010).

\section{TABLE OF CONTENTS LISTING}

The table of contents for the journal will list your paper exactly as it appears below:

Typewriting Dynamics: What Distinguishes Simple From

Complex Writing Tasks?

Sebastian Wallot and Joachim Grabowski 


\title{
Typewriting Dynamics: What Distinguishes Simple From Complex Writing Tasks?
}

\author{
Sebastian Wallot \\ Interacting Minds Centre \\ Aarhus University, Denmark \\ Joachim Grabowski \\ Department of Educational Psychology \\ Leibniz University Hannover, Germany
}

\begin{abstract}
One of the main challenges of writing research has been its generative nature. As a production task, writing cannot be controlled as easily as other psychological laboratory tasks, such as reading. In this article, we present an analysis of 3 writing tasks, ranging from a simplified typing task to an ecological writing task. We show that multiple nonlinear metrics can be used to systematically characterize open, complex writing tasks. Moreover, we demonstrate that, when writing performance is quantified in such a way, a continuum of complexity can be identified on which increasingly complex writing performances can be placed.
\end{abstract}

\section{THE COMPLEXITY OF WRITING PERFORMANCE}

Writing - along with reading, memorizing, visual perception, and simple motor tasks-is one of the pervasive and prominent life-defining activities that has been brought into psychological, physiological, and linguistic laboratories. However, compared with the aforementioned activities, writing has received considerably less scientific attention (see Hayes, 2012, for a recent overview). This is partly

Correspondence should be addressed to Sebastian Wallot, Interacting Minds Centre, Aarhus University, Jens Chr. Skous Vej 4, Building 1.483, 3rd floor, 8000 Aarhus C, Denmark. E-mail: sewa@hum.au.dk 
due to the complex and productive nature of the writing process, which means that it is often impossible to standardize writing in the same way as reading or performance in a simple motor task (Wengelin et al., 2009).

One way to overcome this difficulty is to confine the scientific investigation of writing to certain subtypes of writing task, copy-typing being one of the most prominent examples. However, as Grabowski (2008) identifies, simplified tasks, such as copy-typing, involve different sets of constraints, where many of the core aspects of writing remain inaccessible (Johansson, Wengelin, Johansson, \& 30 Holmqvist, 2010).

To push writing research beyond its current boundaries, two questions have to be addressed: First, how can performance during a complex, productive writing task be quantified systematically? Second, how does performance in simple writing tasks relate to and differ from performance in complex, productive writing tasks?

To address these questions, we present different analyses to investigate the changes in writing performance when experimental control over a task is increasingly relaxed. To that end, we employ three writing tasks used by Grabowski (2008), which are described in more detail in the next section. The three tasks differ in terms of productive opportunities, cognitive involvement, and task constraints, ranging from a simplified and artificial typing task to relatively natural and open text writing.

As the nonlinear dynamics of type-writing are currently unknown, we use several standard statistics (averages and standard deviations) and nonlinear statistics (fractal and recurrence analysis) in conjunction in order to arrive at a detailed description of the performance in the three writing tasks (see Wallot \& Van Orden, 2011). Our aim is to explore which features distinguish best between the tasks and whether the three tasks can indeed be aligned on a dimension that captures increased complexity.

\section{THE WRITING TASKS}

Task 1: Simple typing. Participants were asked to type 12 consecutively numbered repetitions of the first sentence of a well-known German nursery rhyme. Altogether, the text pattern consisted of 531 characters. This task merely involves repetitive "copying" from memory.

Task 2: Copy-typing. Participants were asked to copy a linguistically simple text of 1,170 characters and 156 words. The text, printed on a sheet of paper, remained beside the keyboard. This is a typical copy task.

Task 3: Generation. Participants were asked to type a description of their route from home to the university, as though somebody had asked them for directions. Here, planning and formulation is involved. 
The data gathered from these three typing tasks was expected to reflect the different levels of constraint that each instruction imposed on the task. As participants progressed throughout the tasks, the level of constraint was increasingly relaxed from a very restricted performance in Task 1 to a more 65 natural and open performance in Task 3.

\section{METHOD}

\section{Participants}

Sixty-four trainee teachers from Heidelberg University of Education participated in the experiment, with a mean age of $23.38(S D=1.45)$ years, ranging from $20 \quad 70$ to 28 . None of them had a professional background as a typist. They performed the tasks in individual sessions. It took approximately $30 \mathrm{~min}$ to complete the tasks, and each participant received five Euros compensation.

\section{Apparatus}

The software package ScriptLog 1.8.22 for Windows (Andersson et al., 2006; 75 Strömqvist, Holmqvist, Johansson, Karlsson, \& Wengelin, 2006) was used to record keystroke timing. The basic measures are keystroke intervals, that is, the time between two successive strokes on the keyboard (including characters, function keys, navigation keys, etc.). The ScriptLog text editor window was presented on a standard 17-in. computer screen.

\section{Procedure}

All participants performed the three tasks in the same order (staring with Task 1: simple typing, followed by Task 2: copy-typing, and finally Task 3: generation). Participants were informed that the text editor used for typing functioned in the same way as standard text processing software but without formatting $\mathbf{8 5}$ options.

\section{Data Analysis}

In addition to standard statistics (average and standard deviation), we treated the typing data as time series and quantified their dynamics using fractal and recurrence analyses. These analyses have already been successfully applied to complex text-reading performance (O'Brien, Wallot, Haussmann, \& Kloos, under review; Wallot, O'Brien, \& Van Orden, 2012; Wallot \& Van Orden, 2011) 
and, therefore, it was assumed that they could lend themselves to investigating typing performance.

We used power spectral density to conduct a monofractal analysis, which estimates the degree of long-range correlations across the measured time intervals between keystrokes. A scaling exponent a quantifies the scaling relation between frequency and magnitude of fluctuations. If the scaling exponent is close to 0 , the data series consists of uncorrelated white noise. If the scaling exponent is close to 1 , the data series contains substantial long-range correlations (also referred $\mathbf{1 0 0}$ to as pink noise). Exponents between 0 and 1 correspond to fractional Gaussian noise and quantify the degree of long-range dependencies. Scaling exponents have been found to vary with cognitive and motor skills (Van Orden, Kloos, $\&$ Wallot, 2011) and capture the relative presence of voluntary and involuntary sources of control in cognitive performance (see Kloos \& Van Orden, 2010; Van 105 Orden, 2010).

Multifractal analysis is an extension of monofractal analysis. Whereas monofractal analysis assumes that only a single scaling relation is sufficient to describe a given data set, multifractal analysis investigates the existence of multiple scaling relations that correspond to multiple degrees of fractality in a data set, $\mathbf{1 1 0}$ summarized in a multifractal spectrum. Multifractality is present in the performances of many standard cognitive tasks, such as time estimation, word naming, or lexical decision and indicates online reorganizations of behavior (Ihlen \& Vereijken, 2010). We used continuous wavelet transformation to estimate the multifractal spectrum. Recurrence quantification analysis (RQA) can quantify $\mathbf{1 1 5}$ several dynamic aspects of a time series that are informative regarding its complexity and stability. The central concept of the analysis is that of recurrence, that is, how often does a system repeat its behavior, and how perfect are these repetitions (e.g., Webber \& Zbilut, 2005)?

We report selected recurrence quantifiers that are robust across a wide range of $\mathbf{1 2 0}$ parameter settings in the analyses. The eight quantifications we present capture aspects of time series complexity (phase-space Dimensionality, Entropy) and stability/instability (\%Recurrence, \%Determinism, Meanline, Maxline, \%Laminarity, TrappingTime). A more detailed description of these measures can be found in Webber and Zbilut (2005). ${ }^{1}$

To conduct the analyses, we followed the recommendations in Webber and Zbilut (2005) for RQA, Holden (2005) for monofractal analysis, and Ihlen and Vereijken (2010) for multifractal analysis. We used Marwan's (2011) commandline recurrence plots software to calculate recurrence analysis.

\footnotetext{
${ }^{1}$ To conduct the analysis, the following parameters were used (see Webber \& Zbilut, 2005): Delay $=1$ and Euclidean Norm. The Dimension-parameter was estimated for each data set individually using the False-Nearest-Neighbor algorithm.
} 
Subsequently, we present the results for each of the analyses. To summarize the differences in task performance, a factor analysis was conducted on all the measures used, which is presented after the summary of individual effects, before the Discussion section.

Mean and Standard Deviation Analysis of Key-Press Intervals (KPIs)

As can be seen in Figure 1, we observed an effect of writing task on average KPI $(F(2,126)=3.94, p<.05)$, indicating higher average KPIs for writing during copy-typing compared with the other two writing tasks.

Furthermore, we observed a marginal effect on average standard deviation of 140 KPIs, indicating that copy-typing also yields more widely dispersed KPIs compared with the other two tasks $(F(2,126)=2.59, p=.079)$. However, this marginal effect vanished when the coefficient of variation was used instead $(F<1.64)$.

\section{Monofractal Analysis of Keystroke Intervals}

Analysis of the average scaling exponents revealed reliably higher scaling exponents for copying from memory compared with the other two tasks $(F(2,126)=$ 23.16, $p<.001$ ); see Figure 2.

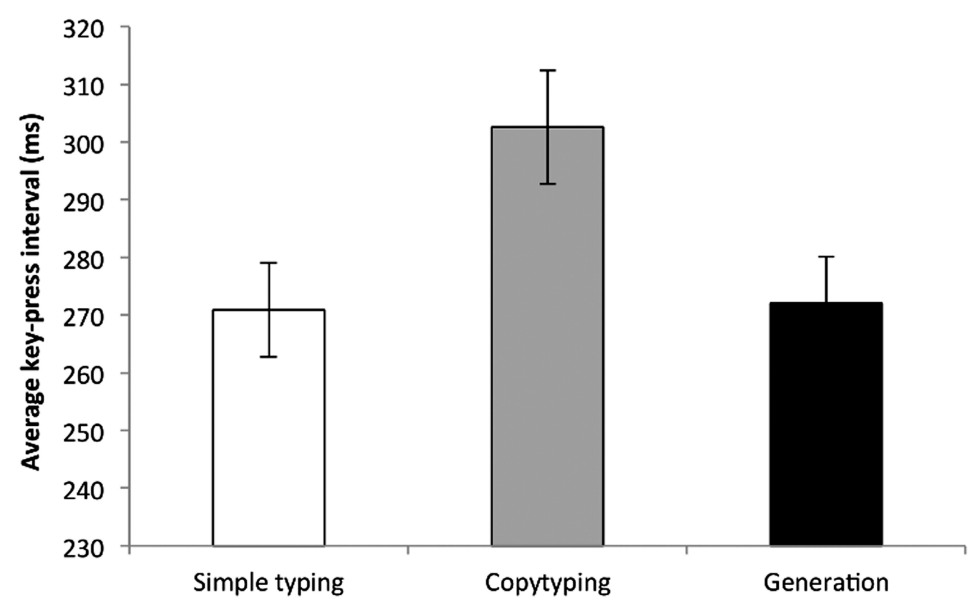

FIGURE 1 Average KPIs bracketed by standard errors of the mean (ms). As can be seen, copy-typing produces longer KPIs compared with the other two tasks. 


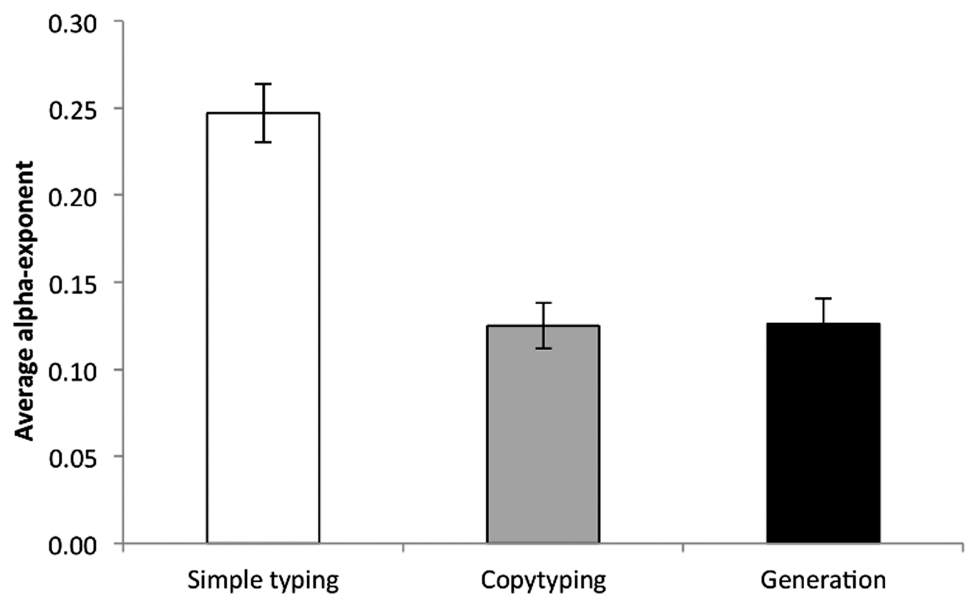

FIGURE 2 Average alpha-exponents bracketed by standard errors of the mean. Alphaexponents are higher for simple typing compared with the other two tasks.

\section{Multifractal Analysis of Keystroke Intervals}

As can be seen in Figure 3, we observed a greater width of the multifractal 150 Fig3 spectrum $(F(2,126)=8.54, p<.001)$ for the generation task (route description) showed a more heterogeneous fractal structure (i.e., a wider multifractal spectrum) compared with copy-typing and simple typing.

\section{Recurrence Quantification Analysis (RQA) of Keystroke Intervals}

Figures 4-7 illustrate the results for Dimension, \%Recurrence, Maxline, and ism, Meanline, TrappingTime, or Entropy (all $F<1.42$ ).

In general, the typing dynamics of copy-typing and generation display higher dimensionality than those of simple typing $(F(2,126)=28.09, p<.001)$; see 160 Figure 4.

Similarly, \%Recurrence $(F(2,126)=7.46, p<.001)$ and \%Laminarity $(F(2,126)=19.08, p<.001)$ increase with task complexity (see Figures 5 and 6).

Maxline yielded only a marginal effect of writing task $(F(2,126),=2.87,165$ $p=.061$, where copy-typing resulted in tentatively higher maximum line length compared with the other two tasks (see Figure 7). 


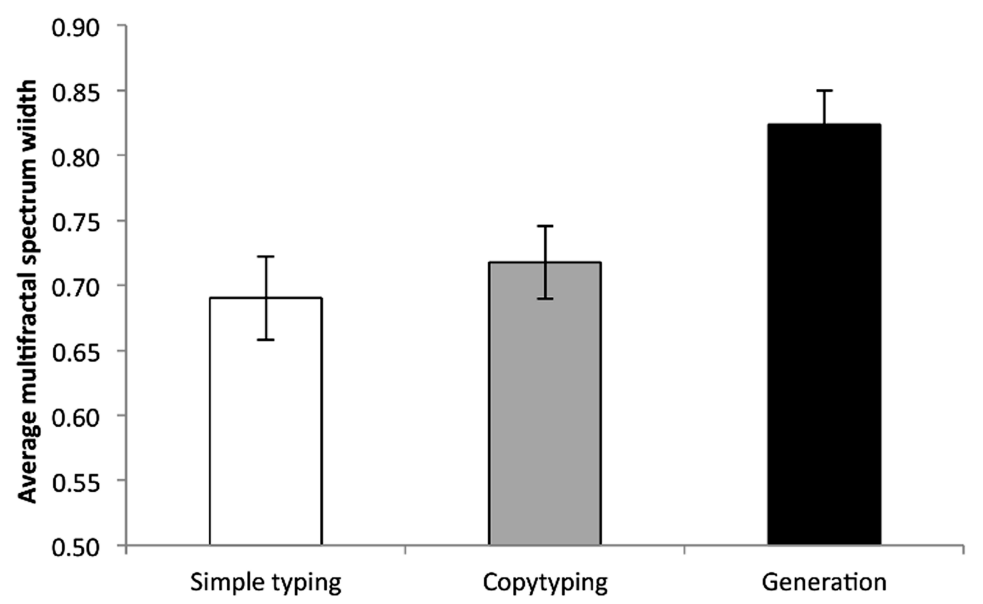

FIGURE 3 Average width of the multifractal spectrum of KPIs by task, bracketed by standard errors of the mean. Multifractal spectrum width is larger for generation task compared with the other two tasks.

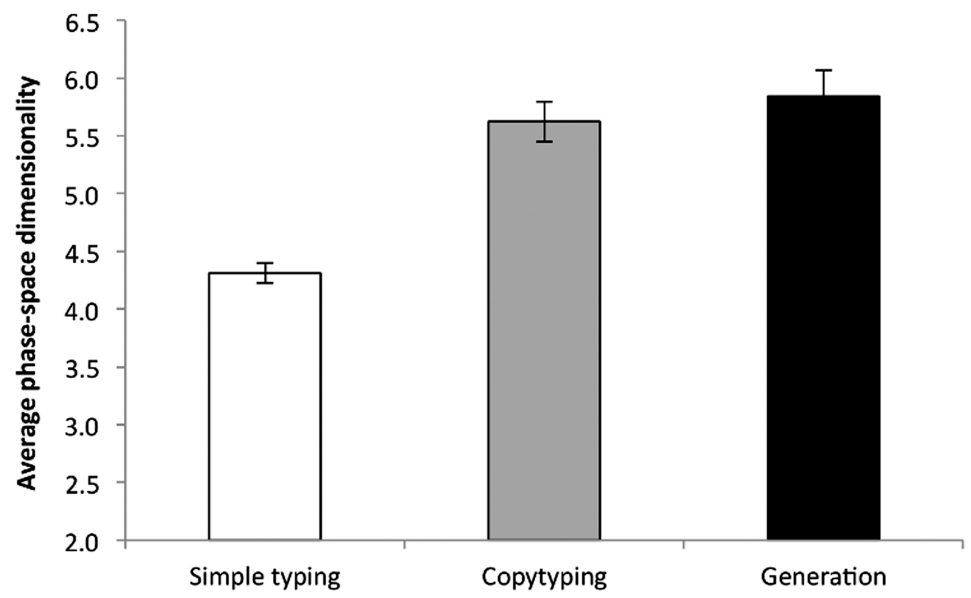

FIGURE 4 Average dimensionality for the typing performance of the three typing tasks bracketed by standard errors of the mean. As can be seen, phase-space dimensionality is lower for simple typing compared with the other two tasks. 


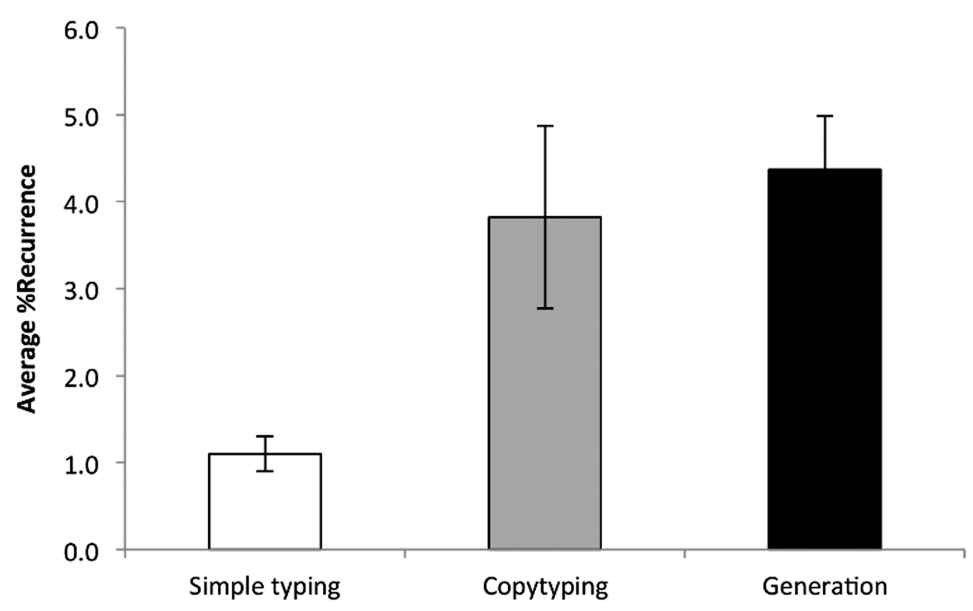

FIGURE 5 Average \%Recurrence by typing tasks, bracketed by standard errors of the mean. \%Recurrence is reliably lower for simple typing compared with the other two tasks.

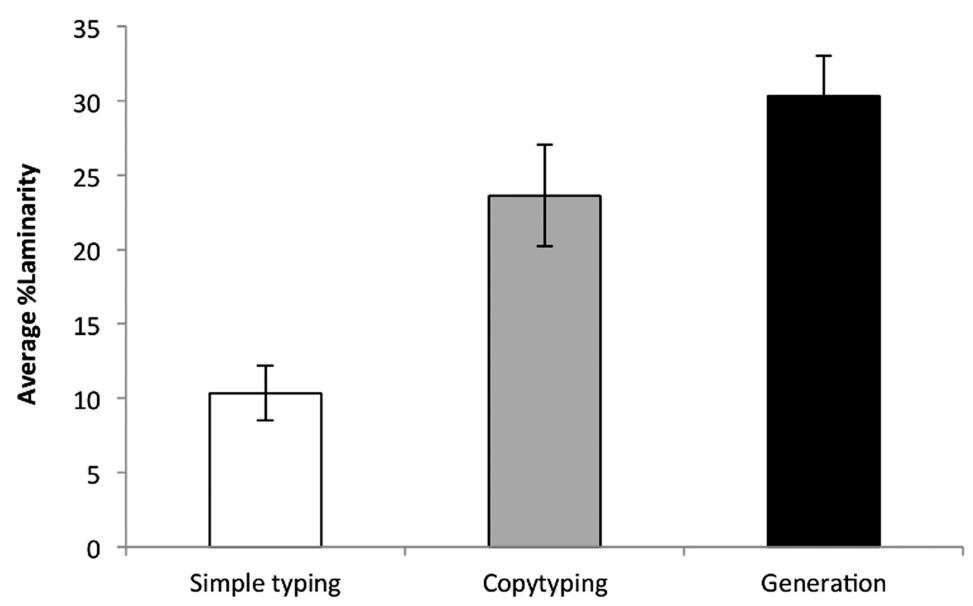

FIGURE 6 Average \%Laminarity by typing task, bracketed by standard errors of the mean. Simple contrasts revealed that \%Laminarity is reliably higher for copy-typing compared with simple typing $(p<.001)$ as well as for generation compared with copy-typing $(p<.05)$. 


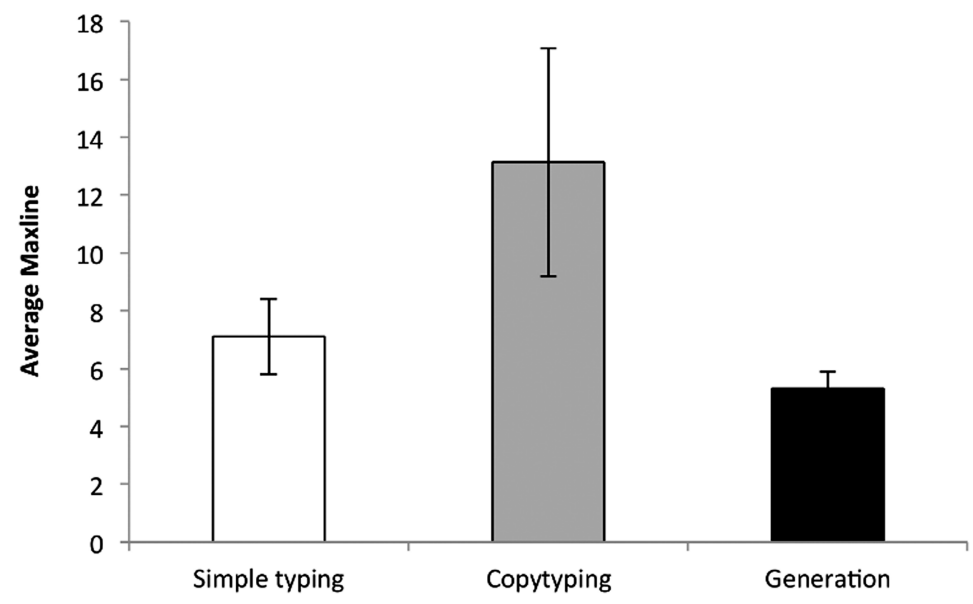

FIGURE 7 Maxline for the typing performance of the three typing tasks, bracketed by standard errors of the mean. Simple contrasts revealed that Maxline is tentatively higher for copy-typing compared with generation $(p=.056)$.

\section{Factor Analysis}

Together with a dummy variable, coded for each of the three tasks, we subjected all variables that distinguished between the three writing tasks to principle $\mathbf{1 7 0}$ Q4 component analysis with varimax rotation. The resulting factor structure and its components are summarized in Table 1 . The overall factor solution yields Tab1 three factors, which account for $62.8 \%$ of the variance of the eight variables.

TABLE 1

Factor Structure of Writing Task Statistics

\begin{tabular}{lrrr}
\hline Variables & Factor 1 & Factor 2 & Factor 3 \\
\hline Task (dummy variable) & $\mathbf{0 . 6 1 8}$ & 0.274 & $\mathbf{0 . 5 3 9}$ \\
Average KPI & -0.246 & $\mathbf{0 . 5 5 8}$ & -0.023 \\
Monofractal PSD & 0.103 & 0.071 & $\mathbf{0 . 8 2 2}$ \\
Multifractal CWT & $\mathbf{0 . 6 1 1}$ & -0.092 & 0.029 \\
Dimensionality & $\mathbf{0 . 8 2 2}$ & -0.046 & -0.080 \\
\%Recurrence & 0.055 & $\mathbf{0 . 8 6 9}$ & 0.068 \\
Maxline & 0.279 & 0.295 & $\mathbf{- 0 . 5 8 5}$ \\
\%Laminarity & $\mathbf{0 . 5 8 0}$ & $\mathbf{0 . 6 7 2}$ & -0.295 \\
Variance explained & $24.0 \%$ & $21.2 \%$ & $17.6 \%$ \\
\hline
\end{tabular}

Note. Substantial contributions to a factor are in boldface. 
Given the moderate sample size (for purposes of factor analysis) of $n=192$ (three data sets for 64 participants), only factor loadings of 0.4 or greater are $\mathbf{1 7 5}$ interpreted as making a substantial contribution to a factor (Sheskin, 2004).

The factors of particular interest to us are those that have substantial commonalities with the dummy variable, which codes for the three writing tasks. As can be seen, there are two factors that covary with the writing tasks: Factors 1 and 3. Both capture aspects of the complexity of typing performance: Factor 180 1 receives contributions from multifractality, phase-space dimensionality, and $\%$ Laminarity. Factor 3 receives contributions from monofractality and Maxline.

Figure 8 is a scatter plot of the factor values of Factor 1 versus Factor 3. As can be seen, both factors separate the three tasks with performance of generation generally scoring high on both factors and performance of simple $\mathbf{1 8 5}$ typing generally scoring low on both factors.

\section{DISCUSSION}

We investigated three writing tasks that manipulated the degree of productivity and cognitive involvement during writing. Simple typing was close to a simple motor task, copy-typing included the need to coordinate perceptual and $\mathbf{1 9 0}$ attentional demands, and generation included productive-cognitive processes. In short, the collation of the different analyses presents the following picture of each task:

Simple typing proceeds in fast typing intervals with a moderate degree of monofractal long-range correlation and a comparatively low degree of multi- 195 fractality. The monofractal structure does indeed move this task closer to simple motor tasks (such as tapping or time estimation), where external, involuntary sources of control (such as a tight coupling between stimulus layout and effector movement) are less prominent. The lower degree of multifractal structure elaborates this observation in that performance is relatively homogeneous, with fewer 200 online-reorganizations of the typing behavior occurring. The smaller phase-space dimensionality corroborates this lower degree of complexity in the KPIs, whereas the low \% Recurrence and \%Laminarity in KPIs indicates that, compared with the two other tasks, typing behavior is also less structured in terms of longer enduring keystroke patterns.

Compared with simple typing, copy-typing resulted in longer keystroke intervals that are close to (monofractal) white noise. Longer (average) key-press intervals are usually associated with more cognitively difficult writing tasks (Wengelin, 2006), but the overall whiter KPIs suggest that typing activity is less due to endogenous motor activity. KPIs during copy-typing are more complex as $\mathbf{2 1 0}$ they "live" in an even higher dimensional phase-space, but this complexity does not appear to stem from reorganizations of the typing process, as multifractality 


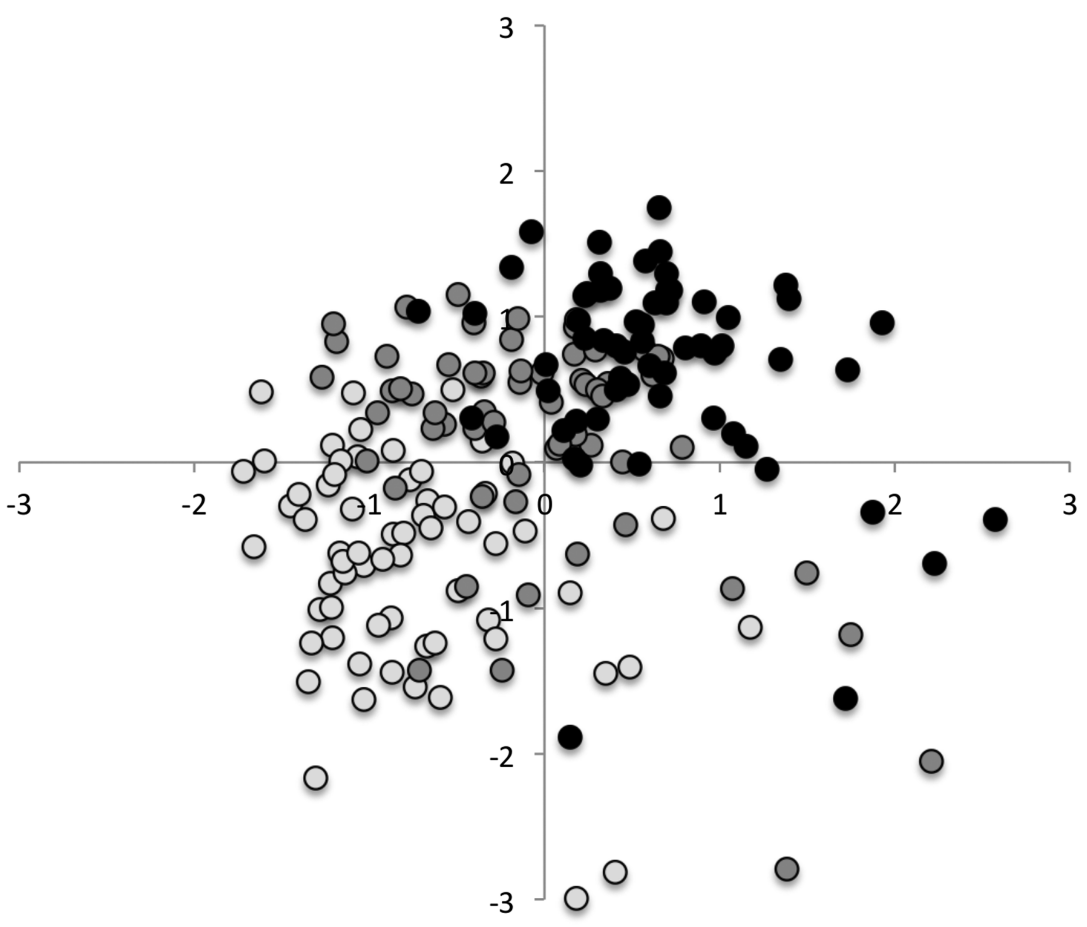

\section{OSimple Typing OCopy-Typing Generation}

FIGURE 8 Scatter plot of Factors 1 and 3 that are related to differences in the writing tasks. White circles display the factor values for simple typing, gray circles display the factor values for copy-typing, and black circles display the factor values for generation. A withinsubject regression analysis (Lorch \& Myers, 1990) revealed a strong positive relationship between the two factors $\left(t(63)=6.77, p<.001, R^{2}=0.44\right)$.

is not reliably increased compared with copying from memory. Therefore, this greater complexity could be a result of the nonrepetitive sequence that lies at the heart of copying a real text instead of repeatedly typing the same pattern. Finally, 215 copy-typing is characterized by a higher \%Recurrence and higher \%Laminarity, which captures the notion that natural typing often proceeds in bursts of keystrokes and not mere isolated keystrokes (Alves, Branco, Castro, \& Olive, 2012).

Finally, for some aspects of the generation task, the observed performance 220 resembles to a greater extent that of copy-typing; keystroke activity is even more complex (higher phase-space dimensionality) than it is in copy-typing, and keystroke activity is even more defined by "bursts" (indicated by higher \%Lam- 
inarity). However, there are also two noticeable differences: first, compared with copy-tying, the average KPIs are somewhat faster and are, in fact, identical to 225 those observed in simple typing. This is remarkable as cognitive involvement in typing is ordinarily considered marked by longer average KPIs, which reflect pauses in writing and indicate cognitive processing (Wengelin, 2006). Moreover, productive writing is arguably the most cognitively complex typing task and should, therefore, produce the longest KPIs (Wengelin et al., 2009). However, 230 what seems to single-handedly distinguish generation from copy-typing (and copying from memory) is the increase in multifractal signature in the keystroke series. This indicates that complex cognitive involvement is captured less well by conventional tenets regarding cognitive processing difficulty but rather by the extent to which qualitative changes during the typing performance are observed. 235

Having characterized the performances of the three typing tasks, the question arises whether these are isolated, distinct tasks or whether they share a certain continuum that maps the decrease of constraint or the increase of "openness" that characterize the tasks. To answer this question, we have conducted a factor analysis of the tasks' distinguishing variables. The analysis yielded three factors $\mathbf{2 4 0}$ (see Figure 8): Factor 2 captures variance from overall length of key-press intervals and from aspects of dynamic stability of key-press times (\%Recurrence and \%Laminarity). This factor is unrelated to the difference between typing tasks, suggesting that key-press latencies of typing performance-which lie at the core of current investigations of writing - do not capture invariants of $\mathbf{2 4 5}$ typing behavior. Similarly, dynamic stability does not seem to be very central in distinguishing between the tasks, although these measures seem to play a core role in reading (O'Brien et al., under review; Wallot et al., 2012; Wallot \& Van Orden, 2011). Perhaps stability is not equally important in "production-oriented" tasks such as typing.

Factors 1 and 3 differentiate between the typing tasks and are more straightforwardly related to complexity: Factor 1 indicates that increasingly complex typing performance goes hand in hand with greater pockets of stable performance (increasing \%Laminarity) but also greater typing-pattern variation (increasing phase-space dimensionality) and drastic reorganizations for typing patterns (in- $\mathbf{2 5 5}$ creasing multifractality). Factor 3 indicates that more complex typing tasks also lead to less rigid (decreasing Maxline) performance, which is more intertwined with involuntary sources of control provided by the task setup (decreasing monofractality).

For the investigation of writing ability and writing performance, this means 260 that even complex writing setups, which allow for open performance, are systematically relatable to more simplified and tightly controlled writing tasks. It also means that this relation is quantifiable by the dynamic characteristics of writing that emphasize the stability and complexity of the organization of the writing process. 
Sebastian Wallot acknowledges funding by the Marie-Curie Initial Training Network, "TESIS: Towards an Embodied Science of InterSubjectivity" (FP7PEOPLE-2010-ITN, 264828).

\section{REFERENCES}

Alves, R. A., Branco, M., Castro, S. L., \& Olive, T. (2012). Effects of handwriting skill: Output 270 modes and gender on fourth graders' pauses, language bursts, fluency and quality. In V. W. Berninger (Ed.), Past, present, and future contributions of cognitive writing research to cognitive psychology (pp. 389-402). New York, NY: Psychology Press.

Andersson, B., et al. (2006). Combining keystroke logging with eye tracking. In L. van Waes, M. Leijten, \& C. Neuwirth (Eds.), Writing and digital media (pp. 166-172). Oxford, UK: Elsevier. 275 Q7

Grabowski, J. (2008). The internal structure of university students' keyboard skills. Journal of Writing Research, 1, 27-52.

Hayes, J. R. (2012). Modeling and remodeling writing. Written Communication, 29, 369-388.

Holden, J. G. (2005). Gauging the fractal dimension of response times from cognitive tasks. In M. A. Riley \& G. C. Van Orden (Eds.), Tutorials in contemporary nonlinear methods for the $\mathbf{2 8 0}$ behavioral sciences: A webbook tutorial (pp. 267-318). Retrieved from http://www.nsf.gov/sbe/ bcs/pac/nmbs/nmbs.jep

Ihlen, E. A. F., \& Vereijken, B. (2010). Interaction-dominant dynamics in human cognition: Beyond 1/f fluctuation. Journal of Experimental Psychology: General, 139, 436-463.

Johansson, R., Wengelin, A., Johansson, V., \& Holmqvist, K. (2010). Looking at the keyboard or the 285 monitor: Relationship with text production processes. Reading and Writing: An Interdisciplinary Journal, 23, 835-851.

Kloos, H., \& Van Orden, G. (2010). Voluntary behavior in cognitive and motor tasks. Mind \& Matter, 8, 19-43.

Lorch, R. F., \& Myers, J. L. (1990). Regression analyses of repeated measures data in cognitive 290 research. Journal of Experimental Psychology: Learning, Memory, and Cognition, 16, 149-157.

Marwan, N. (2011). Commandline recurrence plots. Retrieved from http://www.agnld.uni-potsdam. de/ $\sim$ marwan/6.download/rp.php

O'Brien, B. A., Wallot, S., Haussmann, A., \& Kloos, H. (under review). Using complexity metrics to assess silent reading fluency: A cross-sectional study comparing oral and silent reading.

Sheskin, D. (2004). Handbook of parametric and nonparametric statistical procedures. Boca Raton, FL: Chapman \& Hall.

Strömqvist, S., Holmqvist, K., Johansson, V., Karlsson, H., \& Wengelin, Å. (2006). What keystroke logging can reveal about writing. In K. P. H. Sullivan \& E. Lindgren (Eds.), Computer keystroke logging and writing (pp. 45-71). Oxford, UK: Elsevier.

Van Orden, G. (2010). Voluntary performance. Medicina, 46, 681-594.

Van Orden, G., Kloos, H., \& Wallot, S. (2011). Living in the pink: Intentionality, wellbeing, and complexity. In C. A. Hooker (Ed.), Philosophy of complex systems. Handbook of the philosophy of science. Amsterdam, The Netherlands: Elsevier.

Wallot, S., O'Brien, B. A., \& Van Orden, G. (2012). A tutorial introduction to fractal and recurrence 305 analyses of reading. In G. Liben, G. Jarema, \& C. Westbury (Eds.), Methodological and analytical frontiers in lexical research (pp. 395-430). Amsterdam, The Netherlands: Benjamins.

Wallot, S., \& Van Orden, G. (2011). Nonlinear analyses of self-paced reading. The Mental Lexicon, $6,245-274$. 


\section{WALLOT AND GRABOWSKI}

Webber, C. L., Jr., \& Zbilut, J. P. (2005). Recurrence quantification analysis of nonlinear dynamical 310 systems. In M. A. Riley \& G. C. Van Orden (Eds.), Tutorials in contemporary nonlinear methods for the behavioral sciences (pp. 26-96). Retrieved from http://www.nsf.gov/sbe/bcs/pac/nmbs/ nmbs.jep

Wengelin, Å. (2006). Examining pauses in writing: Theory, methods and empirical data. In K. Sullivan \& E. Lindgren (Eds.), Computer keystroke logging and writing: Methods and applications. 315 Studies in writing (pp. 107-130). Amsterdam, The Netherlands: Elsevier.

Wengelin, Å., Torrance, M., Holqvist, K., Simpson, S., Galbraith, D., Johansson, V., \& Johansson, R. (2009). Combined eyetracking and keystroke-logging methods for studying cognitive processes in text production. Behavioral Research Methods, 41, 337-351. 